\title{
Medulloblastoma, Not Otherwise Specified
}

National Cancer Institute

\section{Source}

National Cancer Institute. Medulloblastoma, Not Otherwise Specified. NCI Thesaurus.

Code C129447.

A medulloblastoma which has not been further characterized. 